\title{
The effect of fatigue on the attentional blink
}

\author{
Jun I. Kawahara $\cdot$ Hirotsune Sato
}

Published online: 11 July 2013

(C) Psychonomic Society, Inc. 2013

It has been suggested that attention and emotional states are tightly linked, in that negative moods are associated with narrowed attentional focus, whereas positive moods are associated with broadened focus. However, recent studies on the effect of affective states, using the attentional blink deficit as a reflection of the temporary unavailability of attentional resources, have reported ambiguous results regarding the deployment of attention in the temporal domain. In the present study, we examined whether the effect of affective state on the attentional blink should be interpreted in terms of valence and arousal axes or in terms of the specificity of the connection between affect and attention. We chose to use fatigue to test these alternatives because, according to the two-axis view, fatigue would not be expected to increase the attentional blink. Participants identified two targets embedded in a stream of nontargets, and for half of the participants, a state of fatigue was induced using the Trier Social Stress Test. Those in the experimental group demonstrated a greater attentional blink relative to those in the control group, who did not receive the mood manipulation. The results suggest unique links between mood states and attention during a task involving temporal selection.

Studies in cognitive psychology have shown that the deployment of attentional resources (Kahneman, 1973; Matthews \& Desmond, 1998) is determined by various external factors, such as physically (Theeuwes, 2010) and socially (West,

\footnotetext{
J. I. Kawahara $(\bowtie)$

Department of Psychology, Chukyo University, 101-2 Yagoto,

Nagoya, Aichi 466-8666, Japan

e-mail: jkawa@lets.chukyo-u.ac.jp

J. I. Kawahara

National Institute of Advanced Industrial Science and Technology,

Tsukuba, Ibaraki, Japan

H. Sato

Shinshu University, Matsumoto, Nagano, Japan
}

Anderson, \& Pratt, 2009) distinctive items, as well as by endogenous factors, such as intention (Hommel, 2010). Moreover, the deployment of attention can also be affected by such internal factors as motivation (Della Libera \& Chelazzi, 2006) and the current mood state (Fredrickson, 2004; Friedman \& Forster, 2010), because the internal states of observers can influence executive function (Ashby, Isen, \& Turken, 1999), which governs the major cognitive systems, thereby including the deployment of attention.

In terms of the spatial domain, it is generally agreed that negative moods are associated with narrowed attentional focus, whereas positive moods are associated with broadened focus (Rowe, Hirsh, \& Anderson, 2007). Therefore, in a typical Eriksen flanker task, in which observers identify a target, the flanking distractors interfered less when participants were in a negative than when they were in a neutral mood (Sato, Takenaka, \& Kawahara, 2012). In contrast, broadened and more distributed attention is more likely in positive than in neutral moods (Moriya \& Nittono, 2011).

Such effects of mood states can also be demonstrated in the temporal domain by using the attentional blink task (Raymond, Shapiro, \& Arnell, 1992), in which identification of the second of two targets embedded in a rapid stream of nontargets is impaired at short intertarget lags (about 100 $300 \mathrm{~ms}$ ). This impairment has been attributed to the unavailability of temporary attentional resources immediately after processing of the first target. Recently, MacLean, Arnell, and Busseri (2010) demonstrated that higher levels of self-reported negative trait affect were associated with a greater attentional blink. These researchers also found that the magnitude of the attentional blink was negatively correlated with trait positive affect and that negative affect was more strongly correlated with the attentional blink than was positive affect, suggesting that negative affect is not simply the absence of positive affect, but rather appears to have its own impact. Moreover, MacLean and Arnell (2010) found that greater dispositional positive affect was associated with 
a smaller attentional blink, whereas greater negative trait affect was associated with a larger attentional blink (see also Rokke, Arnell, Koch, \& Andrews, 2002, for a similar finding related to depression). Therefore, at least chronic negative mood states increase deficits in temporal visual selection.

In terms of acute mood states, the finding that a positive mood produces a smaller attentional blink seems consistent with the results of MacLean et al.'s (2010) study. However, the interpretation was not that simple when negative mood was involved. Specifically, if the principle that holds for chronic states were to apply to acute states, a larger attentional blink should have been observed in the presence of an acute negative mood. In fact, Olivers and Nieuwenhuis (2006) found no impact of negative pictures on the attentional blink, although exposure to a picture with a positive emotional valence significantly reduced the magnitude of the attentional blink, relative to that under the neutral control condition. Schwabe and Wolf (2010) found that increased subjective stress, feelings of unpleasantness, and pain had no impact on the attentional blink.

One possible reason for this inconsistency is that previous studies adopted too simplistic a view, relying on the positive-negative axis to interpret the effect of mood on the attentional blink. A study conducted by Jefferies, Smilek, Eich, and Enns (2008) highlighted this issue. They found that anxiety increased the attentional blink relative to happy and calm mood states, which is consistent with the aforementioned studies showing that negative mood states impair attentional selection. The attentional blink, however, was reduced in participants experiencing a sad mood. On the basis of these results, Jefferies et al. suggested that at least two dimensions are required to interpret the effect of mood states, as is shown in Fig. 1. According to this scheme, the magnitude of the attentional blink is larger in the top left quadrant and smaller in the bottom left quadrant, relative to its magnitude in the right quadrants. However, the same researchers also proposed an alternative explanation: that emotion may be linked to attention through unique connections to specific attributed emotional states, such as sadness, anxiety, and happiness. In other words, the two-axis scheme of valence and arousal may also be too simplistic. Because no supporting evidence for this attractive idea has been provided, the present study was designed to contrast these two alternatives.

In the present study, we examined whether the effect of mood state on the magnitude of the attentional blink should be interpreted in terms of the two axes of valence and arousal or in terms of the specificity of the connection between affect and attention. We manipulated the mood state, fatigue, that best suits the present purpose, as fatigue has been understood as a state with low arousal and almost neutral or slightly negative valence (Feldman Barrett \& Russell, 1998). Therefore, fatigue is located midway between the sad and calm states, as is shown in Fig. 1. If the two-dimensional model of the link between affect and attentional blink is correct, the magnitude of the attentional blink would decrease as the sad mood in the same quadrant of Jefferies et al.'s scheme decreased, or it would remain unchanged, as is the case in the presence of a calm mood. Alternatively, if emotion is linked to attention through connections that are unique to specific attributed emotional states, fatigue would have an impact that differed from those of both sad and calm mood states. We used the Trier Social Stress Test (TSST; Kirschbaum, Pirke, \& Hellhammer, 1993) to induce a fatigue

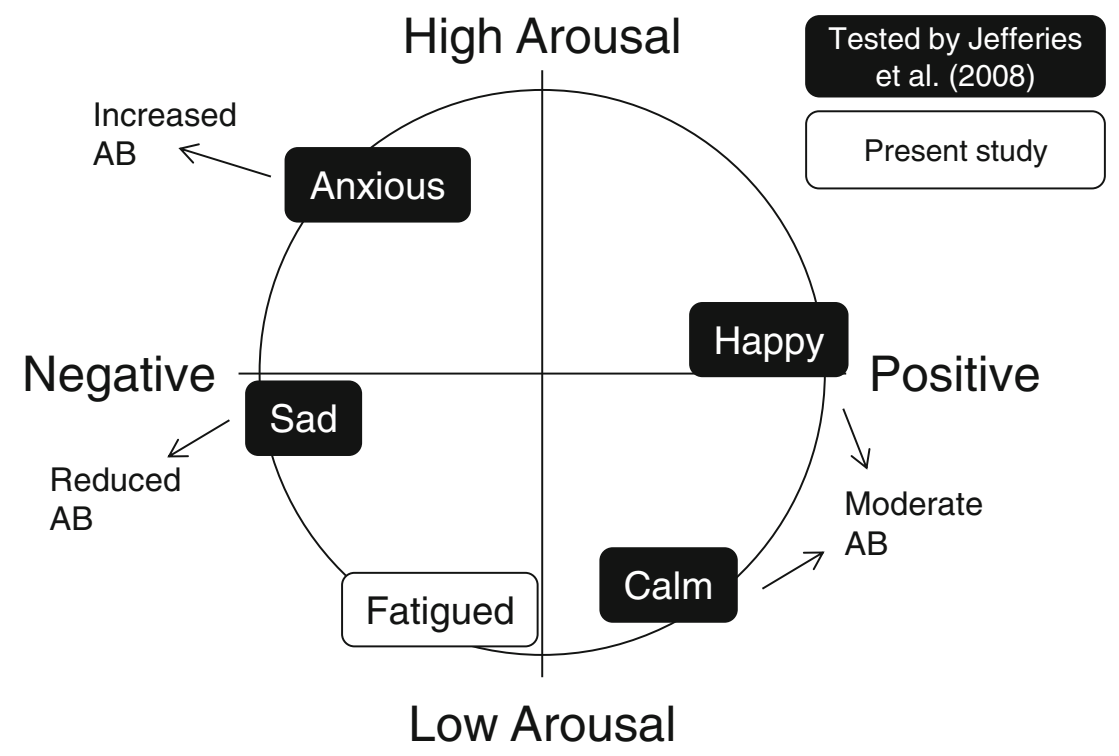

Fig. 1 The four mood states tested by Jefferies et al. (2008), with the resulting modulations of the attentional blink and the fatigue state tested in the present study mapped onto the two-dimensional (i.e., horizontal valence and vertical arousal axes) schematic diagram of affect proposed by Feldman Barrett and Russell (1998) 
state and measured this state using the Profile of Mood States (POMS), which has been shown to provide a reliable and valid measure of fatigue and arousal states (O'Connor, 2004).

\section{Method}

\section{Participants}

A group of 68 participants, recruited from the subject pool of the National Institute of Advanced Industrial Science and Technology (Tsukuba, Japan), participated in exchange for monetary remuneration. The participants were randomly assigned to the experimental $(n=30 ; 15$ female, 15 male, mean age $=24.8$ years $)$ or the control $(n=38 ; 28$ female, 10 male, mean age $=21.0$ years) group. All participants provided written informed consent, and all were tested in the afternoon (between 14:00 and 17:00 h).

\section{Procedure}

Participants completed four tasks: a pretest, the TSST manipulation (experimental group) or filler task (control group), the attentional blink task, and a posttest, in this order.

Pretest measurement Both groups of participants completed the Profile of Mood States (POMS; McNair, Lorr, \& Doppleman, 1971; Yokoyama \& Araki, 1994) and the State-Trait Anxiety Inventory, Form JYZ (STAI; Hidano, Fukuhara, Iwawaki, Soga, \& Spielberger, 2000). The POMS brief form consists of 30 items, each of which is rated on a five-point Likert scale, to assess mood state (tensionanxiety, depression, anger-hostility, vigor, fatigue, and confusion). The STAI consists of 40 items that assess state and trait anxiety. We used only the state-anxiety items (20 items), each of which was rated on a four-point Likert scale. Saliva samples were collected only from the experimental group because of budgetary limitations.

Mood manipulation After completion of the questionnaires, members of the experimental group participated in two stages of socially and cognitively demanding interviews (the TSST procedure). Each participant was instructed to prepare a 5-min speech regarding his or her strengths and weaknesses and to deliver it in front of the two unfamiliar examiners. One of the examiners recorded the speech with a video camera mounted on a tripod located in front of the participant. During the speech, one examiner monitored each participant's nonverbal actions, such as eye-blink frequency and the number of times that he or she looked away or down, using a tally counter and checklist. The other examiner sat in a chair and watched the speech while displaying a sober and reserved attitude. After the speech, the participants completed a 5-min surprise mentalcalculation test in which they serially subtracted 13 from 1,022 as rapidly and as accurately as possible. They were told to restart the subtraction from 1,022 upon each failure. The control group participated in a filler task in which they estimated the duration of a coherent motion or static random-dot patch $\left(2^{\circ} \times 2^{\circ}\right.$ square, filled with 200 dots presented for $\left.1.0-2.5 \mathrm{~s}\right)$ by pressing the spacebar twice so that the interval of the keypress matched the duration of the patch. ${ }^{1}$ This was a pilot experiment intended to identify optimal parameters for use in a different project. The duration of this experiment was approximately the same as that of the experimental manipulation.

Attentional blink task Immediately after the mood manipulation, participants engaged in the attentional blink task. Stimuli were displayed on a computer monitor as black letters and numbers that subtended $1.0^{\circ}$ in height and up to $1.0^{\circ}$ in width. At the beginning of each trial, a central fixation cross $\left(0.4^{\circ}\right.$ in height $\times 0.4^{\circ}$ in width) appeared for $1,000 \mathrm{~ms}$, followed by a 200-ms interval. Then, a stream of nontarget digits was presented in the center of the screen. The stimuli were displayed on a CRT monitor running at $60 \mathrm{~Hz}$. Each appeared for approximately $67 \mathrm{~ms}$ and was followed by a 33-ms interval, yielding a presentation rate of 10 items/s. The nontarget digits were randomly chosen in every trial, under the constraint that the selected digit was not one of the two immediately preceding items. Two different targets - randomly chosen uppercase English alphabet letters, excluding I, O, Q, and Z - were embedded in the stream. The first target appeared between the sixth and twelfth items in the stream with equal probabilities. The intertarget lags were $200,300,400,600$, and $800 \mathrm{~ms}$, and the lag varied randomly within a block. The stream ended with two nontarget digits after the second target. Participants were asked to identify two target letters, regardless of their position in the presentation order, by pressing corresponding keys after the presentation of a stream; no time pressure was imposed. The attentional blink task consisted of ten practice trials followed by 160 experimental trials and lasted approximately $15 \mathrm{~min}$, allowing time for the salivary cortisol response induced by the TSST procedure to

\footnotetext{
${ }^{1}$ To rule out the possibility that this task might have trained the control group in a temporal form of attention, we conducted a control experiment involving two groups of participants $(n=24$ each). One group completed the same coherent-motion task used in the present study, followed by the attentional blink task. The other group completed the same attentional blink task without the coherent-motion task. The accuracy rates of the first group (with the coherent-motion task) were $62.2 \%, 54.1 \%, 58.2 \%, 74.0 \%$, and $73.3 \%$ for lags $1,2,3,5$, and 7 , respectively. The rates of the second group (without the motion task) for the same five lags were $59.9 \%, 60.0 \%, 63.8 \%, 71.2 \%$, and $73.7 \%$, respectively. A two-way ANOVA (Group $\times$ Lag) revealed a main effect of lag, $F(4,184)=18.8, p<.01$, indicating a robust attentional blink effect. We found no effect of group, $F(1,46)=0.01$, n.s., and importantly, no interaction, $F(4,184)=1.42$, n.s. Therefore, the alternative explanation involving training by the control task seems unlikely.
} 
develop and reach measureable levels (Kudielka, Schommer, Helhammer, \& Kirschbaum, 2004) by the end of the task.

Posttest measurement The posttest measurement was identical to the pretest measurement. Both groups completed the POMS and STAI questionnaires for the second time, and saliva samples were collected from the experimental group. Participants were debriefed before their departure.

\section{Results}

\section{Effect of mood manipulation}

The sums of the scores on the POMS subscales and the STAI were calculated and are plotted in Fig. 2 as a function of pre- versus postmanipulation test for each group. A separate 2 (group: experimental vs. control) $\times 2$ (time: pre vs. post) mixed analysis of variance (ANOVA) on the POMS scores was conducted. As expected, the analysis indicated significant effects of the interaction between group and time on vigor $[F(1$, $\left.66)=4.92, p<.05, \eta_{\mathrm{p}}{ }^{2}=.07\right]$ and fatigue $[F(1,66)=6.53$, $\left.p<.05, \eta_{\mathrm{p}}{ }^{2}=.10\right]$. The effect of confusion was also significant $\left[F(1,66)=6.10, p<.05, \eta_{\mathrm{p}}{ }^{2}=.08\right]$. Tests of simple main effects indicated significant effects of time in the experimental group $\left(F_{\mathrm{s}}>6.35, p \mathrm{~s}<.05\right.$; control group: $F_{\mathrm{s}}<3.00$, n.s. $)$, indicating that participants in the experimental group experienced more fatigue and confusion, as well as less vigor, during the second measurement than during the first. With respect to STAI scores, a 2 (group) $\times 2$ (time) mixed ANOVA indicated a significant interaction between group and time, $F(1,66)=4.48$, $p<.05, \eta_{\mathrm{p}}{ }^{2}=.06$. A test of simple main effects revealed a

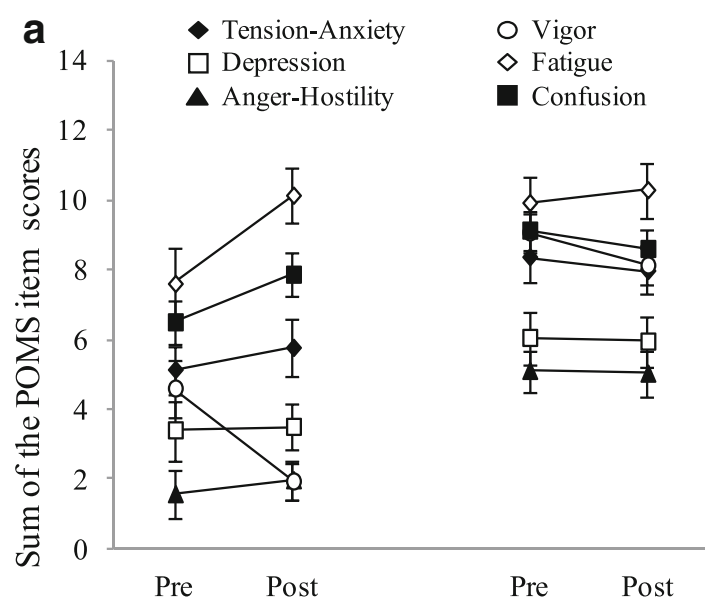

Experimentalgroup Controlgroup

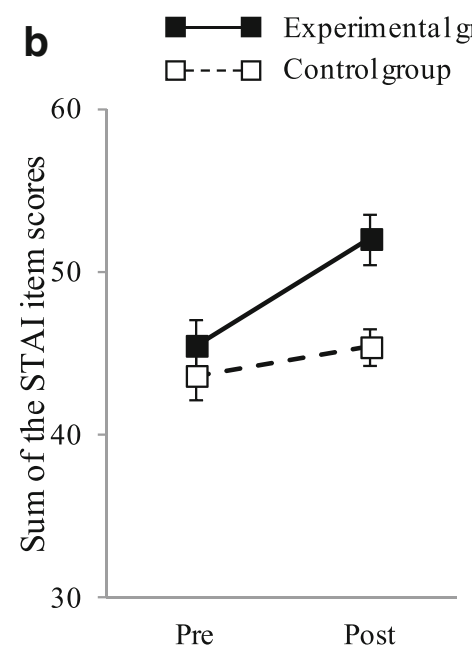

C Experimentalgroup

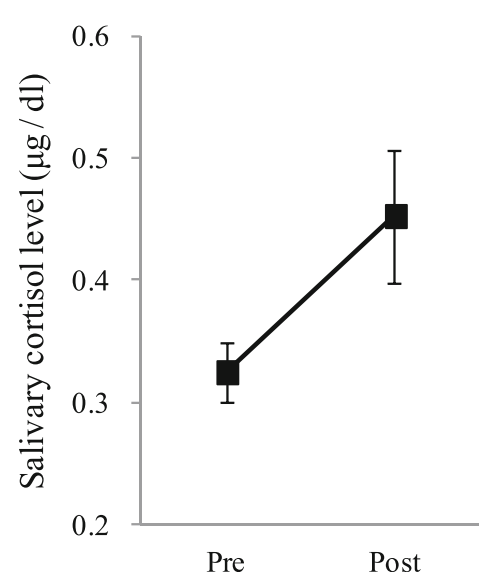

item scores as a function of pre- versus post-stress-manipulation, averaged over the participants in each group. (c) Mean cortisol concentrations in the experimental group as a function of mood manipulation
Fig. 2 (a) Sum of Profile of Mood States (POMS) item scores as a function of pre- versus post-stress-manipulation, averaged over the participants in each group. (b) Sum of State-Trait Anxiety Inventory (STAI) 
significant effect only for time in the experimental group, $F(1$, $66)=16.75, p<.01, \eta_{\mathrm{p}}{ }^{2}=.20$ [control group: $F(1,66)=1.21$, n.s.]

The salivary cortisol levels in 200-ml samples of saliva were assayed with an RIA test (Gamma Coat; Dia Sorin, Stillwater, MN, USA). The results indicated a range of $0.05-$ $3.00 \mu \mathrm{g} / \mathrm{dl}$ and intra-assay coefficients of variation of $<5 \%$ and $<10 \%$ for the pretest and posttest assays, respectively. Figure $2 \mathrm{c}$ presents the cortisol concentrations in the experimental group as a function of the mood manipulation. A $t$ test revealed a significant difference between the pretest and posttest measurements, $t(26)=2.45, p<.05$, Cohen's $d=0.55$.

\section{Attentional blink}

The rates at which the first target was correctly identified averaged across lags under each condition were $88.3 \%$ in the experimental and $90.3 \%$ in the control group. No statistically significant difference was found between these two scores, $t(66)=1.15, p=.29$, n.s. The scores for second-target identification were based only on those trials in which the first target had been correctly identified. Figure 3 shows the rates at which the second target was correctly identified as a function of lag, averaged over all observers under each condition. A two-way ANOVA was conducted with one between-subjects factor (group: experimental vs. control) and one within-subjects factor (lag: $200,300,400,600$, or $800 \mathrm{~ms}$ ). The analysis revealed a significant effect of group, $F(1,66)=4.09, p<.05, \eta_{\mathrm{p}}{ }^{2}=.06$, and lag, $F(4,264)=43.13, p<.01, \eta_{\mathrm{p}}{ }^{2}=.40$. Notably, the interaction was significant, $F(4,264)=2.76, p<.05, \eta_{\mathrm{p}}{ }^{2}=.04$. Tests of the simple main effects revealed lower rates of accurate identifications at shorter lags. Specifically, the rate of accurate

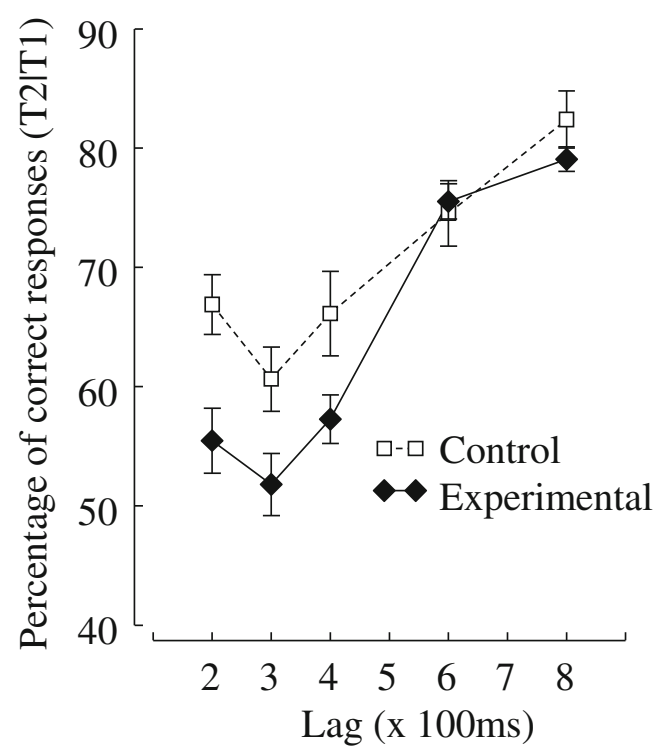

Fig. 3 Percentages of correct identifications of the second target as a function of lag, averaged over all observers under each experimental condition identification was significantly lower in the experimental group than in the control group at a lag of $200 \mathrm{~ms}, F(1,66)=9.83$, $p<.01, \eta_{\mathrm{p}}{ }^{2}=.13$. Similar patterns were observed at $300 \mathrm{~ms}$, $F(1,66)=3.92, p<.10$, and $400 \mathrm{~ms}, F(1,66)=3.17, p<.10$.

To examine the factors related to the modulation of the attentional blink deficit, we calculated the correlations between scores on the questionnaires and the magnitudes of the attentional blink by combining both groups. Magnitude was defined as the difference between the mean second-target accuracy at the two longer lags $(600$ and $800 \mathrm{~ms})$ and that at the three shorter lags $(200,300$, and $400 \mathrm{~ms})$. The analyses revealed that the magnitude of the attentional blink was significantly correlated with the second STAI score, $r=.28, p<.05$, and with the vigor index on the second POMS, $r=-.37, p<.01$. Because the vigor index was scored in reverse, this negative correlation means that less vigor was associated with a greater attentional blink. We also performed partial correlation analyses to control for the pretest scores. The results were virtually the same as in the analysis above: The magnitude of the attentional blink was significantly correlated with the second STAI score, $r_{p}=.27, p=.03$, and with the vigor index on the second POMS, $r=-.39, p=.001$. These results indicated that the difference in the pretest scores of the two groups did not affect the relationship between the mood states and the attentional blink. The present results, however, should be treated with caution, because the correlational analysis involved a small sample that included unequal numbers of men and women, although the effects of sex on responses under these emotional conditions have been mixed (Dickerson \& Kemeny, 2004; Kiecolt-Glaser, Glaser, Cacioppo, \& Malarkey, 1998).

\section{Discussion}

In the present study, we examined whether the effect of mood state on the magnitude of the attentional blink should be interpreted in terms of the two axes of valence and arousal or in terms of the specificity of the connection between affect and attention. We chose fatigue to test these alternatives because fatigue should not increase the attentional blink, according to the two-axis view (Feldman Barrett \& Russell, 1998; Jefferies et al., 2008). The mood manipulation was successful: POMS scores increased at the second measurement, relative to the first, for the dimensions of fatigue and confusion only in the experimental group. Scores for vigor, which was scored in reverse, decreased at the second measurement. No such differences across the two measurements were observed in the control group. Moreover, the salivary cortisol levels of the experimental group increased at the second measurement relative to the first. No systematic differences were found in the control group, which did not receive the mood induction, between the two measurements. 
The POMS scales showed no elevation of anxiety. Therefore, these results indicate that increased fatigue and decreased vigor, rather than anxiety, was induced. A hallmark of the attentional blink was observed, and critically, the magnitude of the attentional blink was greater at shorter lags in the experimental group than in the control group.

Taken together, the present results are consistent with those of previous studies (e.g., Jefferies et al., 2008; MacLean et al., 2010), in that acute affect manipulation modulated the attentional blink. The present results, however, are inconsistent with the two-axis view, proposing that the effect of emotional state on temporal attention can be interpreted in terms of valence and arousal axes. As noted above, we predicted that fatigue would not increase the attentional blink if the two-axis view were correct (Feldman Barrett \& Russell, 1998; Jefferies et al., 2008). This is because fatigue is located in the same quadrant as sadness (see Fig. 1), which reduces the attentional blink, and because no increment in the attentional blink was found by Jefferies et al. when arousal was low. Therefore, the finding that fatigue increased the attentional blink supports the view that emotional states and attention are uniquely linked to specific attributed emotional states. In other words, the twoaxis scheme of valence and arousal may be too simplistic. The present results also provide indirect support for Schwabe and Wolf's (2010) study, which showed that stress had no effect on the attentional blink, given the unique link between affect and attention.

What are the implications of the present results for recent models of the attentional blink? The present data can be integrated into one of the two classes of attentional blink models that have postulated that this phenomenon reflects a limitation in the resources available for consolidating the second target into working memory (e.g., Chun \& Potter, 1995; Kessler et al., 2005). Because various negative mood states impair the encoding of events into working memory (e.g., Schoofs, Wolf, \& Smeets, 2009), it is reasonable to assume that the attentional blink would be increased in the presence of those moods. The second class of models, which attribute the attentional blink to the selection (Nieuwenstein \& Potter, 2006) or the control (Di Lollo, Kawahara, Ghorashi, \& Enns, 2005; Olivers \& Meeter, 2008; Taatgen, Juvina, Schipper, Borst, \& Martens, 2009) process, do not explicitly introduce the effect of mood states. Thus, no specific predictions can be derived from these models. Although Olivers and Nieuwenhuis (2006) interpreted variance in the magnitude of the attentional blink in terms of the dependence of the distribution of attentional resources on valence, the present results suggest that endorsement of such a simplistic view is premature. Consistent with this argument, Huntsinger (2012) has provided evidence challenging the idea that the valence dimension alone shapes attentional scope.

In conclusion, the present study has demonstrated that a sociocognitive mood manipulation detrimentally affected the temporal selection and encoding of targets presented among briefly presented nontargets. Specifically, the TSST stress manipulation increased the magnitude of the attentional blink deficit. The present affect modulation of the attentional blink cannot be explained in terms of a simple two-axis view. Although it is less parsimonious, the view that emotional states and attention are linked uniquely to specific attributed emotional states was supported by our data (Jefferies et al., 2008). Obviously, no universally accepted framework for affect exists. Thus, further investigation using a different type of affect will be necessary to test whether the view of unique affect-attention links is viable and to examine whether frameworks other than the two-axis view (e.g., Plutchik, 2001) could better explain the temporal modulation of attention by affective states.

\section{References}

Ashby, F. G., Isen, A. M., \& Turken, A. U. (1999). A neuropsychological theory of positive affect and its influence on cognition. Psychological Review, 106, 529-550. doi:10.1037/0033-295X.106.3.529

Chun, M. M., \& Potter, M. C. (1995). A two-stage model for multiple target detection in rapid serial visual presentation. Journal of Experimental Psychology. Human Perception and Performance, 21, 109-127. doi:10.1037/0096-1523.21.1.109

Della Libera, C., \& Chelazzi, L. (2006). Visual selective attention and effects of monetary rewards. Psychological Science, 17, 222-227. doi:10.1111/j.1467-9280.2006.01689.x

Di Lollo, V., Kawahara, J., Gorashi, S. M., \& Enns, J. T. (2005). The attentional blink: Resource depletion or temporary loss of control? Psychological Research, 69, 191-200. doi:10.1007/s00426-004-0173-x

Dickerson, S. S., \& Kemeny, M. E. (2004). Acute stressors and cortisol responses: A theoretical integration and synthesis of laboratory research. Psychological Bulletin, 130, 355-391.

Feldman Barrett, L., \& Russell, J. A. (1998). Independence and bipolarity in the structure of current affect. Journal of Personality and Social Psychology, 74, 967-984.

Fredrickson, B. L. (2004). The broaden-and-build theory of positive emotions. Philosophical Transactions of the Royal Society B, 359, 1367-1377.

Friedman, R. S., \& Förster, J. (2010). Implicit affective cues and attentional tuning: An integrative review. Psychological Bulletin, 136, 875-893. doi:10.1037/a0020495

Hidano, T., Fukuhara, M., Iwawaki, M., Soga, S., \& Spielberger, C. D. (2000). State-Trait Anxiety Inventory (Form JYZ) test manual (Japanese adaptation of STAI). Tokyo, Japan: Jitsumu Kyoiku Shup-pan.

Hommel, B. (2010). Grounding attention in action control: The intentional control of selection. In B. J. Bruya (Ed.), Effortless attention: A new perspective in the cognitive science of attention and action (pp. 121-140). Cambridge, MA: MIT Press.

Huntsinger, J. R. (2012). Does positive affect broaden and negative affect narrow attentional scope? A new answer to an old question. Journal of Experimental Psychology. General, 141, 595-600.

Jefferies, L. N., Smilek, D., Eich, E., \& Enns, J. T. (2008). Emotional valence and arousal interact in attentional control. Psychological Science, 19, 290-295. doi:10.1111/j.1467-9280.2008.02082.x 
Kahneman, D. (1973). Attention and effort. Englewood Cliffs, NJ: Prentice-Hall.

Kessler, K., Schmitz, F., Gross, J., Hommel, B., Shapiro, K., \& Schnitzler, A. (2005). Cortical mechanisms of attention in time: Neural correlates of the lag-1-sparing phenomenon. European Journal of Neuroscience, 21, 2563-2574.

Kiecolt-Glaser, J. K., Glaser, R., Cacioppo, J. T., \& Malarkey, W. B. (1998). Marital stress: Immunologic, neuroendocrine, and autonomic correlates. Annals of the New York Academy of Sciences, 840, 656-663.

Kirschbaum, C., Pirke, K. M., \& Hellhammer, D. H. (1993). The "Trier Social Stress Test"-A tool for investigating psychobiological stress responses in a laboratory setting. Neuropsychobiology, 28, 76-81.

Kudielka, B. M., Schommer, N. C., Hellhammer, D. H., \& Kirschbaum, C. (2004). Acute HPA axis responses, heart rate, and mood changes to psychosocial stress (TSST) in humans at different times of day. Psychoneuroendocrinology, 29, 983-992.

MacLean, M. H., \& Arnell, K. M. (2010). Personality predicts temporal attention costs in the attentional blink paradigm. Psychonomic Bulletin \& Review, 17, 556-562. doi:10.3758/PBR.17.4.556

MacLean, M. H., Arnell, K. M., \& Busseri, M. A. (2010). Dispositional affect predicts temporal attention costs in the attentional blink paradigm. Cognition and Emotion, 24, 1431-1438.

Matthews, G., \& Desmond, P. A. (1998). Personality and multiple dimensions of task-induced fatigue: A study of simulated driving. Personality and Individual Differences, 25, 443-458.

McNair, D., Lorr, M., \& Doppleman, L. (1971). POMS manual for the Profile of Mood States. San Diego, CA: Educational and Industrial Testing Service.

Moriya, H., \& Nittono, H. (2011). Effect of mood states on the breadth of spatial attentional focus: An event related potential study. Neuropsychologia, 9, 1162-1170. doi:10.1016/j.neuropsychologia. 2011.02.036

Nieuwenstein, M. R., \& Potter, M. C. (2006). Temporal limits of selection and memory encoding: A comparison of whole versus partial report in rapid serial visual presentation. Psychological Science, 17, 471-475. doi:10.1111/j.1467-9280.2006.01730.x

O'Connor, P. J. (2004). Evaluation of four highly cited energy and fatigue mood measures. Journal of Psychosomatic Research, 57, 435-441.

Olivers, C. N. L., \& Meeter, M. (2008). A boost and bounce theory of temporal attention. Psychological Review, 115, 836-863. doi:10. 1037/a0013395
Olivers, C. N. L., \& Nieuwenhuis, S. (2006). The beneficial effects of additional task load, positive affect, and instruction on the attentional blink. Journal of Experimental Psychology. Human Perception and Performance, 32, 364-379. doi:10.1037/00961523.32.2.364

Plutchik, R. (2001). The nature of emotion. American Scientist, 89, 344-350.

Raymond, J. E., Shapiro, K. L., \& Arnell, K. M. (1992). Temporary suppression of visual processing in an RSVP task: An attentional blink? Journal of Experimental Psychology. Human Perception and Performance, 18, 849-860. doi:10. 1037/0096-1523.18.3.849

Rokke, P. D., Arnell, K. M., Koch, M. D., \& Andrews, J. T. (2002). Dual-task attention deficits in dysphoric mood. Journal of Abnormal Psychology, 111, 370-379.

Rowe, G., Hirsh, J. B., \& Anderson, A. K. (2007). Positive affect increases the breadth of attentional selection. Proceedings of the National Academy of Sciences, 104, 383-388.

Sato, H., Takenaka, I., \& Kawahara, J. (2012). The effects of acute stress and perceptual load on distractor interference. Quarterly Journal of Experimental Psychology, 65, 617-623.

Schoofs, D., Wolf, O. T., \& Smeets, T. (2009). Cold pressor stress impairs performance on working memory tasks requiring executive functions in healthy young men. Behavioural Neuroscience, 123, 1066-1075. doi:10.1037/a0016980

Schwabe, L., \& Wolf, O. T. (2010). Emotional modulation of the attentional blink: Is there an effect of stress? Emotion, 10, 283288.

Taatgen, N. A., Juvina, I., Schipper, M., Borst, J. P., \& Martens, S. (2009). Too much control can hurt: A threaded cognition model of the attentional blink. Cognitive Psychology, 59, 129.

Theeuwes, J. (2010). Top-down and bottom-up control of visual selection. Acta Psychologica, 135, 77-99. doi:10.1016/j.actpsy.2010. 02.006

West, G. L., Anderson, A. A. K., \& Pratt, J. (2009). Motivationally significant stimuli show visual prior entry: Evidence for attentional capture. Journal of Experimental Psychology. Human Perception and Performance, 35, 1032-1042.

Yokoyama, K., \& Araki, S. (1994). POMS Japanese manual (in Japanese). Tokyo, Japan: Kaneko Syobo. 